\title{
Automatic Arteriovenous Ratio Computation: Emulating the Experts
}

\author{
S.G. Vázquez ${ }^{1}$, N. Barreira ${ }^{1}$, M.G. Penedo ${ }^{1}$, M. Rodriguez-Blanco ${ }^{2}$, F. Gómez-Ulla ${ }^{2}$, \\ A. González ${ }^{1}$, and G. Coll de Tuero ${ }^{3}$ \\ ${ }^{1}$ Varpa Group, Department of Computer Science, University of A Coruña, Spain \\ ${ }^{2}$ Service of Ophthalmology, Complejo Hospitalario Univ., Santiago de Compostela, Spain \\ ${ }^{3}$ Research Unit. Health Care Institute, Girona, Spain \\ \{sgonzalezv, nbarreira, mgpenedo\} @udc.es, \\ \{mariarodriguez, gomezulla\}@itogalicia.es, \\ ana.gonzalez@udc.es, gcolld@meditex.es
}

\begin{abstract}
The arteriovenous ratio is an objective way to assess the arteriolar narrowing related to several diseases such as hypertension. It is computed as the ratio between the artery and vein mean widths. However, its calculus is not straightforward since the experts do not use all the retinal vessels. This paper presents an automatic, precise and reproducible methodology for the AVR computation. We analyze the way the experts select the vessels in order to build a system which emulates them. The system was evaluated by two ophthalmologists in a data set of 86 images. The correlation results among the system and the experts are an indication of the reproducibility of the results.
\end{abstract}

Keywords: arteriovenous ratio computation, retinal imaging, vessel segmentation, vessel classification.

\section{Introduction}

The retinal analysis is a key field for the early diagnosis of several diseases due to retinal blood vessels can be observed directly in a non-invasive way. So, many medical studies explore the relationship between macro- and retinal micro-circulation and associate the width of retinal blood vessels with cardiovascular diseases such as hypertension, strokes, and diabetes $[1,2]$. For example, arteriolar narrowing is considered an initial damage in vascular diseases, especially in hypertension. However, the European guidelines for the management of arterial hypertension explicitly exclude arteriolar narrowing to stratify the cardiovascular risk of hypertension due to its subjectivity, imprecision and poor reproducibility. In this sense, some efforts have been made to develop precise and reproducible methods to measure the degree of narrowing in arteries. On this point, an objective way to assess the arteriolar narrowing is the arteriovenous ratio (AVR), this is, the ratio between arteriolar and venular vessel widths. However, the AVR estimation is a difficult task. It involves measuring and classifying the vessels in an area concentric to the optic disc and deciding which ones are appropriate for the calculus. Despite these difficulties, ophthalmologists continue estimating this measure in clinical trials obtaining high correlation values between them although they do not select the same vessel subset. 
Several computer-assisted methods have been proposed $[1,3,4]$ to achieve an accurate and reproducible measurement. These methods still require user assistance in some phases such as classification of vessels into arteries and veins, the location of the optic nerve or the selection of the suitable vessels for the computation. Recently, some automatic approaches have been presented [5]. However, the validation in large data sets and the testing of its prognosis capacity are currently pending tasks.

We had developed a web-based system [6] for retinal image analysis which includes a service to compute the AVR in a semiautomatic way. In that system, once the experts have selected the optic disc position, the system detects the vessels in several circumferences centered at the optic nerve and measures their widths. After that, the experts select the suitable vessels for the AVR computation, classifying them into arteries or veins. Finally, the AVR is calculated as the ratio between the averages of the artery and vein widths. In this paper, we present an improvement to compute the AVR automatically. The new application does not require the user assistance for the optic disc location and the vessel classification tasks so it is fully automatic. In this work, we are mainly concerned on analyzing how different experts select the vessels to achieve a system which emulates the medical experts.

The remaining of the paper is structured as follows. Section 2 explains the proposed methodology to compute the AVR automatically. Section 3 evaluates the system performance. Finally, in Section 4, we provide some concluding remarks.

\section{Method}

We propose a methodology to compute the AVR automatically that comprises several stages. First, the optic nerve is located and then the vessels are segmented and measured in several circumferences concentric to the optic disc. After that, the vessel segments obtained are classified as arteries and veins. Finally, the system selects an appropriate set of vessel segments to estimate the AVR.

\subsection{Optic Disc Location}

The optic nerve location algorithm is based on the Hough transform [7]. First, the region of interest is delimited using a blob detection method, the Difference of Gaussians operator. In this manner, we convolve the original image with two Gaussians filters at different scales and we obtain the difference between these convolutions. The first approximation of the optic disc center is taken as the maxima of DoG output. The input of this transform is the set of edge pixels computed from the original image. However, only the optic disc edges are necessary. Thus, we combine the Sobel operator and the MLSEC-ST [8] outputs in order to discard other edges, such as vessel edges. Finally, we extract the optic disc from the remaining edges using the Hough transform.

\subsection{Measurement of Vessel Widths}

We have proposed a method to measure automatically the vessel widths in several circumferences centered on the optic disc [9]. That method is based on snakes. A snake is a contour defined within the image that evolves in order to fit some features 
of interest. This evolution is governed by external and internal energies in such a way that the model reaches a minimum when it fits the features of interest. In our case, these features are the vessel boundaries. To this end, we initialize a snake in the intersection points of each circumference and the vessel centerlines. The vessel centerlines are detected by means of the MLSEC-ST operator [8]. Each snake evolves in the direction perpendicular to the centerlines and stops at the vessel contours detected by the Sobel operator. Once the evolution stops, a topological adjustment is performed in order to avoid deviations in the snake contour. Finally, the vessel widths are measured from the snake contours.

\subsection{Vessel Classification into Artery and Vein}

Medical experts use color information to classify arteries and veins. However this task is not straightforward. Although veins are darker than arteries, differences are only visible in main vessels. In the case of secondary vessels, the ophthalmologists have to follow the vessel tree from the optic nerve.

A methodology to classify the retinal vessels has been proposed [10] and it has been improved in [11]. This method involves a local classification to avoid the influence of the lightness variability and the lack of color constancy, and a global integration of local color differences to ensure the decision. First, the multi-scale retinex algorithm is applied to original image to enhance the color constancy. Next, we divide the image into four quadrants centered at the optic disc and we apply a clustering algorithm in each quadrant. Then, the axis is rotated $20^{\circ}$ and the classification is performed again in each new quadrant. This process is repeated until it achieves a $180^{\circ}$ degree rotation. Finally, the classification results are combined to obtain the final class of a vessel segment. After that, we joint the local classification using a tracking strategy [11] which connects the vessel segments which belong to the same vessel in different circumferences.

\section{$2.4 \quad$ Vessel Selection}

In the semiautomatic approach [6], once the medical experts had classified the vessels into arteries and veins, the AVR is computed as the ratio between the artery and vein average widths. However, the experts do not take into account all the detected vessels but only a subset of them. They follow some unwritten rules to select the most suitable vessels and, although two experts do not select exactly the same subset, the correlation between them is high [12]. This way, we analyze a large data set of images where the AVR was computed by two experts in order to obtain some hypothesis of the selection rules used by experts. Thus, after a knowledge acquisition process and an analysis of the vessel selection made by experts, we have obtained the following hypothesis:

H1: Experts rule out the vessels whose width has been over- or sub-estimated.

$\mathrm{H} 2$ : Experts do not take into account vessel segments found over a bifurcation or a crossover.

H3: Experts do not select the same number of arteries and veins at each analysis radius.

H4: Experts select the same global number of arteries and veins. 
H5: Experts do not take into account thin vessels, either because it is more difficult to know their classes or because it is more probable to overestimate their width. H6: Experts rule out false positive vessels detected in the background.

We have implemented an algorithm of vessel selection taking into account the above selection hypothesis. The H1 hypothesis has been implemented using the tracking algorithm. To rule out the vessels with over or sub-estimated width, we obtain the median width of each connected segment and we rule out the vessel segments whose width is larger than the double or smaller than the half of the median width. In addition, these extreme vessels can be also detected by means of percentiles, discarding the vessels whose widths are less than the p percentile or larger than the 100-p percentile.

A method which detects bifurcation and crossovers [13] has been applied to implement the $\mathrm{H} 2$ hypothesis. In this case, we have two options, since we can remove the segments before or after the classification procedure. Removing them before the classification can be an advantage in case of crossovers because we avoid altering the mean color value used in the vessel classification procedure since we do not know which vessel is on top of the crossover.

Hypothesis $\mathrm{H} 3$ and $\mathrm{H} 4$ were carried out excluding the smallest arteries and veins until the number of vessel segments in both classes are the same, globally or in each circumference, respectively.

Hypothesis H5 is performed in three different ways. Two of them consist on ruling out the vessels by percentiles. First, we remove the vessels whose widths are smaller than the $\mathrm{p}$ percentile. Another way is computing two different percentiles, one for veins and the other for arteries. The third manner involves detecting fewer vessels, using more restrictive parameters in the MLSEC-ST operator.

We eliminate all the isolated vessel segments which are not connected to any other to carry out the last hypothesis, H6. Moreover, the number of false positives is also reduced when the parameters of the MLSEC-ST operator become more restrictive.

In addition, according to other authors, we try another selection method which just consists of taking into account the six main arteries and six main veins to estimate the AVR. In this case, we only consider the 12 main vessels with the largest median width along the vessel.

\subsection{AVR Computation}

The arteriovenous ratio can be computed of three different ways $[1,14,15]$. In our case, we estimate the AVR as the ratio between the average of artery widths and the average of vein widths $[4,14]$.

\section{Results}

The proposed methodology has been tested on a set of eighty six color retinal images acquired with a Cannon CR6-45NM non-mydriatic retinal camera at the hospital of Conxo, Santiago de Compostela (Spain). These images have a resolution of $768 \times 576$ pixels. 
Table 1. Test hypothesis results between experts and/or automatic system

\begin{tabular}{cccc}
\hline Experts & $\begin{array}{c}\text { Pearson's correlation } \\
\text { coefficient }\end{array}$ & $\begin{array}{c}\text { p-value of } \\
\text { Pearson's test }\end{array}$ & $\begin{array}{c}\text { p-value of Welch's } \\
\text { t-test }\end{array}$ \\
\hline Expert1 vs. Expert2 & 0.7981 & $<2.2 \mathrm{e}-16$ & 0.2496 \\
Expert1 vs. System ${ }_{\mathrm{H} 1 \mathrm{H} 5}$ & 0.7466 & $<2.2 \mathrm{e}-16$ & 0.8641 \\
${\text { Expert2 vs. System } \mathrm{H}_{\mathrm{H} 5 \mathrm{H} 2}}$ & 0.7940 & 0 & 0.0138 \\
Expert2 vs. System $_{\mathrm{H} 1 \mathrm{H} 5}$ & 0.7860 & 0 & 0.34 \\
\hline & $\mathbf{6}$ main vessels \\
\hline Expert1 vs. Expert2 & 0.8546 & $<2.2 \mathrm{e}-16$ & $<2.2 \mathrm{e}-16$ \\
Expert1 vs. System & 0.7472 & $<2.2 \mathrm{e}-16$ & 0.6036 \\
Expert2 vs. System & 0.7161 & $9.104 \mathrm{e}-15$ & 0.3464 \\
\hline
\end{tabular}
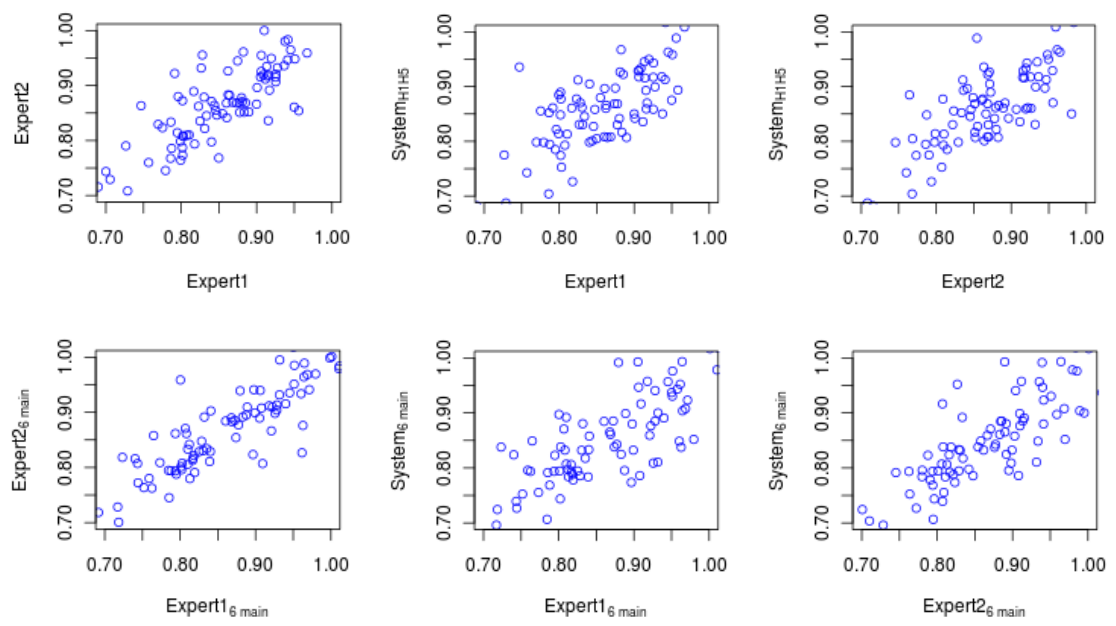

Fig. 1. Dispersion graphs that show the dependence between the AVR measured by different experts

Two ophthalmologists with many years of experience in the study of microvasculature involvement in hypertensive and diabetic retinopathy have estimated the AVR in each fundus image using the automatic system correcting the wrong artery/vein classifications, changing the automatic optic disc location and/or eliminating the vessels that they consider unsuitable for the AVR computation. A Pearson's correlation coefficient of 0.7981 , as well as, a non significative hypothesis whose null hypothesis is that the coefficient is different than zero, indicate that there is a high correlation between experts (see Table 1). Moreover, a Welch's t-test was also performed and the p-value achieved reveals that the AVR's estimated by experts are equal in average. If we only take into account the 6 main arteries and veins labeled by experts to estimate the AVR, the correlation increases. The evaluation of the vessel 
selection algorithm involved testing 649 combinations of hypothesis. Table 1 summarizes the results achieved. It shows the three highest Pearson's correlation coefficients among each expert and the system when the Welch's t-test resulted non significative. Figures 1 and 2 show dispersion and boxplot graphs of the AVR estimated by the different experts.

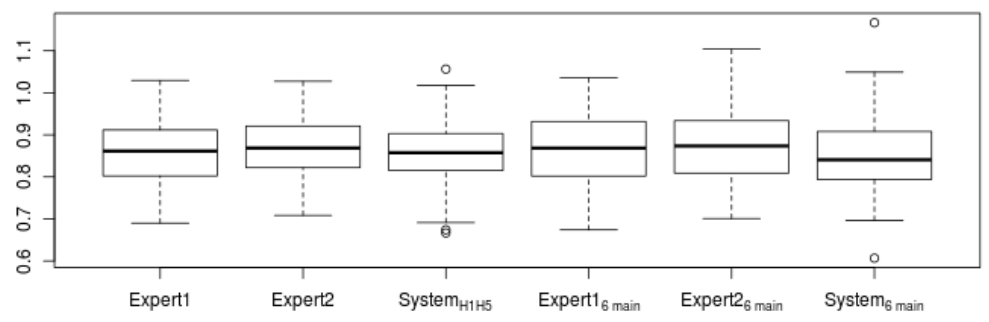

Fig. 2. Boxplot of AVR values estimated by different experts. System ${ }_{\mathrm{H} 1 \mathrm{H} 5}$ denotes the most correlated AVR between both experts and the system.

In general, the best options for the vessel selection algorithm are those which implement the $\mathrm{H} 1, \mathrm{H} 5$, and $\mathrm{H} 2$ hypothesis. Hypothesis $\mathrm{H} 5$ has two different implementations but the correlation results were similar. The best results were obtained eliminating those arteries and veins whose width is smaller than the 10percentiles computed for each class. In addition, if we only take into account the 6 main vessels, the correlation between the each expert and the system is lower.

\section{Conclusions}

In this work, we analyze several alternatives for the vessel selection stage in order to develop an automatic AVR computation system. Taking a semi-automatic AVR computation framework as a starting point, the proposed selection procedure provides an estimation of the AVR close to the estimation measured by medical experts in a quite large data set. Therefore, we can say that the automatic system behaves almost like other expert. However, our analysis shows that the selection of vessels has a high influence on the AVR value and that the best selection procedure includes discarding thin vessels, over- and sub-estimated widths as well as segments over bifurcations or crossovers.

Establishing guidelines for the selection of vessels is necessary but it seems a challenge. For that reason, the AVR should be computed using the same subset of vessel segments for each patient in order to use this variable as a reliable prognosis value. This way, future work in this field includes the development of a monitoring methodology using the same vessel points. 


\section{References}

1. Hubbard, L.D., Brothers, R.J., King, W.N., Clegg, L.X., Klein, R., Cooper, L.S., Sharrett, A.R., Davis, M.D., Cai, J.: Methods for evaluation of retinal microvascular abnormalities associated with hypertension/sclerosis in the atherosclerosis risk in communities studies. Ophthalmology 106, 2269-2280 (1999)

2. Wong, T.Y., Klein, R., Klein, B.E.K., Tielsch, J.M., Hubbard, L., Javier Nieto, F.: Retinal microvascular abnormalities and their relationship with hypertension, cardiovascular disease, and mortality. Survey of Ophthalmology 46(1), 59-80 (2001)

3. Li, H., Hsu, W., Lee, M.L., Wong, T.Y.: Automatic grading of retinal vessel width. Heart 52(7), 1352-1355 (2009)

4. Pose-Reino, A., Gómez-Ulla, F., Hayik, B., Rodríguez-Fernández, M., Carreira-Nouche, M.J., Mosquera-González, A., González-Penedo, M., Gude, F.: Computerized measurement of retinal blood vessel calibre: description, validation and use to determine the influence of ageing and hypertension. Journal of Hypertension 23(4), 843-850 (2005)

5. Tramontan, L., Grisan, E., Ruggeri, A.: An improved system for the automatic estimation of the arteriolar-to-venular diameter ratio (avr) in retinal images. In: 30th Annual International IEEE EMBS Conference, Vancouver, British Columbia, Canada, pp. 3550 3553 (2008)

6. Ortega, M., Barreira, N., Novo, J., Penedo, M.G., Pose-Reino, A., Gómez-Ulla, F.: Sirius: A web-based system for retinal image analysis. International Journal of Medical Informatics 79, 722-732 (2010)

7. Blanco, M., Penedo, M.G., Barreira, N., Penas, M., Carreira, M.J.: Localization and Extraction of the Optic Disc Using the Fuzzy Circular Hough Transform. In: Rutkowski, L., Tadeusiewicz, R., Zadeh, L.A., Żurada, J.M. (eds.) ICAISC 2006. LNCS (LNAI), vol. 4029, pp. 712-721. Springer, Heidelberg (2006)

8. Lopez, A.M., Lloret, D., Serrat, J., Villanueva, J.J.: Multilocal creaseness based on the level-set extrinsic curvature. Computer Vision and Image Understanding 77, 111-144 (2000)

9. Caderno, I.G., Penedo, M.G., Barreira, N., Mariño, C., González, F.: Precise Detection and Measurement of the Retina Vascular Tree. Pattern Recognition and Image Analysis: Advances in Mathematical Theory and Applications (IAPC Nauka/Interperiodica) 15(2), 523-526 (2005)

10. Vázquez, S.G., Barreira, N., Penedo, M.G., Ortega, M., Pose-Reino, A.: Improvements in retinal vessel clustering techniques:towards the automatic computation of the arteriovenous ratio. Computing, Archives for Scientific Computing 90(3), 197-217 (2010)

11. Vázquez, S.G., Cancela, B., Barreira, N., Penedo, M.G., Saez, M.: On the automatic computation of the arterio-venous ratio in retinal images: Using minimal paths for the artery/vein classifications. In: International Conference on Digital Image Computing: Techniques and Applications (DICTA 2010), pp. 599-603 (2010)

12. Pose-Reino, A., Pena Seijo, M., González Penedo, M., Ortega Hortas, M., Rodríguez, M.: Estimation of the retinal microvascular calibre in hypertensive patients with the snakes semiautomatic model. Med Clin (Barc) 135(4), 145-150 (2010) 
13. Ortega, M., Rouco, J., Novo, J., Penedo, M.G.: Vascular Landmark Detection in Retinal Images. In: Moreno-Díaz, R., Pichler, F., Quesada-Arencibia, A. (eds.) EUROCAST 2009. LNCS, vol. 5717, pp. 211-217. Springer, Heidelberg (2009)

14. Stanton, A., Mullaney, P., Fainsia, M., O’Brien, E., O'Malley, K.: A method of quantifying retinal microvascular alterations associated with blood pressure and age. Journal of Hypertension 13(1), 41-48 (1995)

15. Michael, D., Knudtson, K.E., Lee, L.D.: Revised formulas for summarizing retinal vessel diameters. Current Eye Research 27(3), 143-149 (2003) 